\title{
OTIMIZAÇÃO DOS PARÂMETROS DE OPERAÇÃO NO PROCESSO DE COAGULAÇÃO/FLOCULAÇÃO E SEDIMENTAÇÃO NO TRATAMENTO DE ÁGUA COM A ASSOCIAÇÃO PAC E Moringa oleifera Lam
}

\author{
Karina Cardoso Valverde ${ }^{1}$ \\ Priscila Ferri Coldebella ${ }^{2}$ \\ Letícia Nishi ${ }^{3}$ \\ Tássia Rhuna Tonial dos Santos ${ }^{4}$ \\ Onélia Aparecida Andreo dos Santos ${ }^{5}$ \\ Rosângela Bergamasco ${ }^{6}$
}

\begin{abstract}
Resumo: Existem vários estudos sobre coagulantes naturais, contudo, informações sobre as condições de operação no processo de coagulação/ floculação e sedimentação ainda são insuficientes, principalmente relacionadas aos efeitos dos coagulantes químicos em conjunto com a Moringa oleifera Lam (M. oleifera). Assim, o objetivo foi avaliar a eficiência da associação dos coagulantes policloreto de alumínio (PAC) e M. oleifera na remoção de cor aparente, turbidez e compostos com absorção em $U^{254 n m}\left(U_{254 n m}\right)$, em função da alteração das velocidades e tempos de mistura, a fim de se obter as condições de operações ideais para água bruta com turbidez inicial de 72,9 NTU. Os ensaios foram realizados em Jar Test, com água bruta proveniente da bacia do rio Pirapó, Maringá, PR, com dosagens de $12,5 \mathrm{mg} . \mathrm{L}^{-1}$ para o PAC associado a $50 \mathrm{mg} . \mathrm{L}^{-1}$ de M. oleifera. Para a avaliação da remoção dos parâmetros de qualidade utilizou-se delineamento fatorial $27 \times 4$, sendo vinte e sete variações quanto à velocidade de mistura rápida e lenta (VMR e VML) e tempos de mistura rápida e lenta (TMR e TML); e quatro tempos de sedimentação (TS). Por meio da aplicação do teste Tukey, observou-se que as condições de operação mais eficientes foram: $100 \mathrm{rpm}$ (VMR), $1 \mathrm{~min}$ (TMR), $45 \mathrm{rpm}$ (VML), $15 \mathrm{~min}$ (TML e TS), com eficiência de remoção de $97,1 \%, 95,8 \%$ e $85,6 \%$ para cor aparente, turbidez e $\mathrm{UV}_{254 \mathrm{~nm}}$, respectivamente.
\end{abstract}

Palavras-chave: Coagulação/floculação, condições de operação, Moringa oleifera Lam, policloreto de alumínio, tratamento de água.

Abstract: There are several studies on natural coagulants, however, informations about the conditions of operation in the coagulation/flocculation and sedimentation process is still insufficient, mainly related to the effects of chemical coagulants associating with Moringa oleifera Lam ( $M$. oleifera). Thus, this study aims to evaluate the efficiency of the association of coagulants polyaluminium chloride (PAC) and $M$. oleifera in the removal of apparent color, turbidity, and compounds with absorption in $\mathrm{UV}_{254 \mathrm{~nm}}\left(\mathrm{UV}_{254 \mathrm{~nm}}\right)$, in light of changing speeds and mixing times, in order to obtain the optimal conditions for operations with initial raw water turbidity of 72.9 NTU. The tests were performed in Jar Test, with raw water from the river Pirapó, Maringá, PR, with dosages of $12.5 \mathrm{mg} . \mathrm{L}^{-1}$ for PAC associated with $50 \mathrm{mg} . \mathrm{L}^{-1} \mathrm{M}$. oleifera. To assess the removal of the quality parameters we used factorial design $27 \times 4$, being twenty-seven variations in the speed of fast and slow mixing (VMR and VML) and times of rapid mixing and slow (TMR and TML) and four times sedimentation (TS). By applying the Tukey test it was observed that the most interesting operating conditions were: $100 \mathrm{rpm}$ (VMR), $1 \mathrm{~min}$ (TMR), $45 \mathrm{rpm}$ (VML), 15

\footnotetext{
${ }^{1}$ Universidade Estadual de Maringá, UEM. Email: karinacvalverde@gmail.com

${ }^{2}$ Universidade Estadual de Maringá, UEM. Email: priscila.ferri@bol.com.br

${ }^{3}$ Universidade Estadual de Maringá, UEM. Email: leticianishi@ hotmail.com

${ }^{4}$ Universidade Estadual de Maringá, UEM. Email: tassia_tonial@ hotmai.l.com

${ }^{5}$ Universidade Estadual de Maringá, UEM. Email: onelia@ deq.uem.br

${ }^{6}$ Universidade Estadual de Maringá, UEM. Email: rosangela@deq.uem.br
} 
min (TML and TS) with removal efficiency of $97.1 \%, 95.8 \%$ and $85.6 \%$ for color apparent, turbidity and $\mathrm{UV}_{254 \mathrm{~nm}}$, respectively.

Keywords: Coagulation/floculation, operation conditions, Moringa oleifera Lam, polyaluminium chloride, water treatment.

\section{INTRODUÇÃO}

A utilização de coagulantes para a remoção de cor, turbidez, matéria orgânica e demais impurezas presentes na água, têm sido utilizada com sucesso no tratamento para a produção de água potável.

Nas Estações de Tratamento de Águas (ETAs), o fenômeno da coagulação, começa a ocorrer de maneira simultânea na mistura rápida, e se consolida durante a mistura lenta (floculação).

De acordo com Pritchard et al. (2010), uma mistura rápida de poucos segundos é importante após a adição de um coagulante para assegurar uma dispersão uniforme e promover o contato entre as partículas presentes na água bruta.

A floculação é uma das operações unitárias baseada em fenômenos físicos, que visa reduzir o número de partículas presentes na massa líquida. $\mathrm{O}$ tempo de mistura lenta auxilia na formação dos flocos.

$\mathrm{Na}$ etapa de sedimentação, aos flocos formados são fornecidas condições que os permitam depositar pela ação da gravidade, resultando na clarificação do sobrenadante.

Nos últimos anos, a utilização de vários coagulantes naturais tem sido estudada como uma opção ao tratamento de água convencional e a Moringa oleifera Lam ( $M$. oleifera) se destaca como um promissor coagulante natural (Amagloh e Benang, 2009; Madrona et al., 2010; Pritchard et al., 2010; Vijayaraghavan et al., 2011; Poumaye et al., 2012).

Esta planta pode ser facilmente propagada por adaptar-se em locais com pluviometria baixa e climas quentes. Não tem exigências quanto ao tipo de solo
(Gidde et al. 2012), só mostrando impossibilidade de se desenvolver em temperaturas abaixo de $8^{\circ} \mathrm{C}$ e períodos de geadas (Poumaye et al., 2012).

Nwaiwu et al. (2012) afirmam que, as sementes apresentam o melhor potencial de coagulação/floculação. Supõe-se que essas são utilizadas como coagulante na clarificação de água devido à presença de uma proteína coagulante catiônica solúvel capaz de reduzir a turbidez da água tratada (Pritchard et al., 2010; Gidde et al., 2012).

Poumaye et al. (2012) avaliaram que a $M$. oleifera pode ser utilizada na clarificação da água com eficiência elevada. Amagloh e Benang (2009) recomendam em casos de baixa eficiência de remoção, a associação da $M$. oleifera com outro coagulante para se obter uma água de melhor qualidade.

Diversos pesquisadores já investigaram o comportamento da $M$. oleifera como auxiliar de coagulação no tratamento de água, principalmente com sais de alumínio (Abaliwano et al., 2008; Bina et al., 2010; Fatombi et al., 2012; Rodrígues-Núñes et al., 2012; Valverde et al. 2013a). Contudo, Vijayaraghavan et al. (2011) afirmam que, ainda existe uma escassez de estudos abrangentes que comparam a eficiência da associação dos coagulantes natural e químicos.

Apesar da existência de muitas pesquisas sobre a utilização de coagulantes naturais, informações sobre as condições de operação no processo de coagulação/ floculação ainda são insuficientes, principalmente relacionadas aos efeitos dos coagulantes químicos em conjunto com a $M$. oleifera.

Como os parâmetros de mistura e sedimentação influenciam na eficiência de remoção das impurezas contidas na água bruta, é interessante então estudá-los por meio da realização de experimentos 
em Jar Test, a fim de definir condições adequadas de coagulação/floculação.

Segundo Baghvand et al. (2009), os parâmetros de mistura, incluindo o tempo e intensidade, podem afetar a eficiência de remoção de turbidez da água. Cardoso et al. (2008) comprovaram que o tempo para propiciar a mistura rápida, mistura lenta e sedimentação, influenciam no processo de coagulação/ floculação e sedimentação.

Assim, este estudo propõe avaliar a eficiência da associação dos coagulantes PAC e M. oleifera na remoção de cor aparente, turbidez e compostos com absorção em $U_{254 n m}\left(U_{254 n m}\right)$, em função da alteração dos tempos e velocidades de mistura, a fim de se obter as condições de operações ideais para o processo de coagulação/floculação da água bruta.

\section{MATERIAIS E MÉTODOS}

Os ensaios de coagulação/ floculação e sedimentação foram realizados no Laboratório de Gestão, Controle e Preservação Ambiental, do Departamento de Engenharia Química (DEQ), da Universidade Estadual de Maringá (UEM), utilizando a água bruta coletada na Companhia de Saneamento do Paraná (SANEPAR). As amostras de água são provenientes da bacia do rio Pirapó, Maringá, Paraná, Brasil.

A caracterização inicial da água bruta foi realizada por meio dos seguintes parâmetros de qualidade: cor aparente e $\mathrm{UV}_{254 \mathrm{~nm}}$ (espectrofotômetro DR 5000 Hach), turbidez (turbidímetro 2100P Hach), carbono orgânico dissolvido (COD) (analisador de carbono orgânico total TOC-L CPH Shimadzu), sólidos dissolvidos totais (SDT) (APHA, 1995) e
pH (pHmetro Thermo-Scientific VSTAR92 Orion Versastar).

\subsection{Preparação dos coagulantes}

Para a preparação da solução padrão do PAC foi considerada uma concentração de $1 \% \mathrm{v} / \mathrm{v}$, ou seja, para cada $1 \mathrm{~mL}$ de PAC, completou-se o volume para $100 \mathrm{~mL}$ com água destilada. Para a obtenção do coagulante em pó integral, $5 \mathrm{~g}$ de sementes de $M$. oleifera provenientes de Aracaju, Sergipe, Brasil, foram descascadas manualmente, trituradas em liquidificador doméstico e secas em estufa com circulação e renovação de ar (Digital Timer SX $\mathrm{CR} / 42$ ) a $40^{\circ} \mathrm{C}$ até peso constante (Amagloh e Benang, 2009).

\subsection{Condições de operação}

As condições a serem avaliadas na associação dos coagulantes foram determinadas a partir das condições de operação ótimas da $M$. oleifera, estudadas por Cardoso et al. (2008) e Madrona et al. (2012); e as condições de operação para o PAC foram determinadas a partir das condições reais de operação da SANEPAR.

Para as condições de mistura rápida, variou-se as velocidades (VMR) de $100 \mathrm{rpm}$ a $110 \mathrm{rpm}$ durante o tempo (TMR) de 1 min a 3 min. Para as condições de mistura lenta, variou-se as velocidades (VML) entre $15 \mathrm{rpm}$ e 45 rpm durante o tempo (TML) de $15 \mathrm{~min}$, conforme apresentado na Tabela 1 (Valverde et al., 2013b).

As amostras de água tratada foram coletadas no tempo de sedimentação (TS) de $15 \mathrm{~min}, 30 \mathrm{~min}, 45 \mathrm{~min}$ e $60 \mathrm{~min}$. 
Tabela 1. Condições de Operação Avaliadas para o Processo de Coagulação/Floculação com a Associação PAC e M. oleifera para Tratamento de Água Bruta

\begin{tabular}{|c|c|c|c|c|c|c|c|c|c|c|c|c|c|c|c|}
\hline Ensaios & 1 & 2 & 3 & 4 & 5 & 6 & 7 & 8 & 9 & 10 & 11 & 12 & 13 & 14 & 15 \\
\hline VMR (rpm) & 100 & 105 & 110 & 100 & 105 & 110 & 100 & 105 & 110 & 100 & 105 & 110 & 100 & 105 & 110 \\
\hline TMR (min) & 1 & 1 & 1 & 2 & 2 & 2 & 3 & 3 & 3 & 1 & 1 & 1 & 2 & 2 & 2 \\
\hline VML (rpm) & 15 & 15 & 15 & 15 & 15 & 15 & 15 & 15 & 15 & 30 & 30 & 30 & 30 & 30 & 30 \\
\hline TML (min) & 15 & 15 & 15 & 15 & 15 & 15 & 15 & 15 & 15 & 15 & 15 & 15 & 15 & 15 & 15 \\
\hline
\end{tabular}

\begin{tabular}{|c|c|c|c|c|c|c|c|c|c|c|c|c|}
\hline Ensaios & 16 & 17 & 18 & 19 & 20 & 21 & 22 & 23 & 24 & 25 & 26 & 27 \\
\hline VMR (rpm) & 100 & 105 & 110 & 100 & 105 & 110 & 100 & 105 & 110 & 100 & 105 & 110 \\
\hline TMR (min) & 3 & 3 & 3 & 1 & 1 & 1 & 2 & 2 & 2 & 3 & 3 & 3 \\
\hline VML (rpm) & 30 & 30 & 30 & 45 & 45 & 45 & 45 & 45 & 45 & 45 & 45 & 45 \\
\hline TML (min) & 15 & 15 & 15 & 15 & 15 & 15 & 15 & 15 & 15 & 15 & 15 & 15 \\
\hline
\end{tabular}

\subsection{Processo de Coagulação/ Floculação e Sedimentação}

Os ensaios de coagulação/ floculação foram realizados em Jar Test simples Nova Ética, Modelo 218/LDB06 de seis provas, com regulador de rotação das hastes misturadoras em recipientes com capacidade para $700 \mathrm{~mL}$ de água bruta.

A temperatura da água foi mantida na faixa de $25,0 \pm 3,0^{\circ} \mathrm{C}$ em todos os ensaios (Cardoso et al., 2008).

As dosagens adicionadas de coagulantes nos ensaios foram adaptadas segundo valores citados na literatura, sendo $12,5 \mathrm{mg} . \mathrm{L}^{-1}$ para o PAC (Makki et al., 2010) e $50 \mathrm{mg} . \mathrm{L}^{-1}$ para a $M$. oleifera (Joshua e Vasu, 2013).

Após o processo de coagulação/ floculação e sedimentação, $15 \mathrm{~mL}$ de água tratada foi coletada, e a avaliação da eficiência do processo foi realizada com base na redução percentual dos parâmetros cor aparente, turbidez e $\mathrm{UV}_{254 \mathrm{~nm}}$.

\subsection{Análise estatística}

Para a avaliação da remoção dos parâmetros de qualidade de água cor aparente, turbidez e $\mathrm{UV}_{254 \mathrm{~nm}}$, após os processos de coagulação/floculação e sedimentação, utilizou-se delineamento fatorial $27 \mathrm{x} 4$, sendo os fatores: ensaios (vinte e sete variações quanto às VMR,
TMR, VML e TML) e tempos de sedimentação (quatro TS), com duas repetições (Valverde et al., 2013b).

Foram realizados para comparação dos resultados a Análise de Variância (ANOVA) e o teste de comparação de médias, teste Tukey, com 95\% de confiança, sendo significativo um p-valor $<0,05$, para verificar as diferenças significativas das eficácias de remoção dos parâmetros avaliados, através do programa estatístico SISVAR versão 5.3.

\section{RESULTADOS E DISCUSSÃO}

A Tabela 2 apresenta os resultados da caracterização inicial da água bruta utilizada nos ensaios.

Tabela 2. Caracterização da Água Bruta

\begin{tabular}{|c|c|c|}
\hline Parâmetro & Unidade & Valores \\
\hline Cor aparente & $\mathrm{uH}$ & 386 \\
\hline Turbidez & $\mathrm{NTU}$ & 72,9 \\
\hline $\mathrm{UV}_{254 \mathrm{~nm}}$ & $\mathrm{~cm}^{-1}$ & 0,275 \\
\hline $\mathrm{COD}$ & $\mathrm{mg} \cdot \mathrm{L}^{-1}$ & 3,410 \\
\hline $\mathrm{SDT}$ & $\mathrm{mg} \cdot \mathrm{L}^{-1}$ & 71 \\
\hline $\mathrm{pH}$ & - & 7,813 \\
\hline
\end{tabular}

As condições de operação que apresentaram as melhores eficiências de remoção dos parâmetros cor aparente, turbidez e $U V_{254 n m}$ estão apresentadas na Tabela 3. 
Tabela 3. Valores das Eficiências de Remoção para as Melhores Condições de Operação na Associação PAC e M. oleifera

\begin{tabular}{|c|c|c|c|c|c|c|c|}
\hline \multicolumn{1}{c|}{ Parâmetros } & \multicolumn{5}{c|}{ Eficiências de remoção (\%) } \\
\hline $\begin{array}{c}\text { VMR } \\
(\mathbf{r p m})\end{array}$ & $\begin{array}{c}\text { TMR } \\
(\mathbf{m i n})\end{array}$ & $\begin{array}{c}\text { VML } \\
(\mathbf{r p m})\end{array}$ & $\begin{array}{c}\text { TML } \\
(\mathbf{m i n})\end{array}$ & $\begin{array}{c}\text { TS } \\
(\mathbf{m i n})\end{array}$ & $\begin{array}{c}\text { Cor } \\
\text { aparente }\end{array}$ & Turbidez & $\mathbf{U V}_{\mathbf{2 5 4 n m}}$ \\
\hline 105 & 2 & 30 & 15 & $45-60$ & $94,1-94,5$ & 94,8 & $81,0-81,2$ \\
\hline 110 & 2 & 30 & 15 & $45-60$ & $95,0-95,1$ & $93,7-93,8$ & $81,9-82,1$ \\
\hline 100 & 3 & 30 & 15 & 45 & 95,4 & 93,6 & 81,3 \\
\hline 110 & 3 & 30 & 15 & 60 & 95,8 & 93,6 & 82,8 \\
\hline 100 & 1 & 45 & 15 & $15-60$ & $95,9-97,4$ & $95,7-96,0$ & $84,8-85,6$ \\
\hline 105 & 1 & 45 & 15 & $15-30 / 60$ & $96,3-97,3$ & $95,7-96,1$ & $83,8-85,1$ \\
\hline 110 & 1 & 45 & 15 & $15-60$ & $95,4-96,0$ & $95,3-96,0$ & $82,8-84,6$ \\
\hline 100 & 2 & 45 & 15 & $30-60$ & $95,1-95,5$ & $95,2-95,5$ & $82,1-83,1$ \\
\hline 100 & 3 & 45 & 15 & $45-60$ & $95,2-96,2$ & $95,0-95,6$ & $83,1-84,2$ \\
\hline 105 & 3 & 45 & 15 & $15-45$ & $95,4-96,2$ & $95,8-96,4$ & $82,3-84,4$ \\
\hline 110 & 3 & 45 & 15 & $15-60$ & $95,0-95,7$ & $96,0-96,4$ & $81,5-82,9$ \\
\hline
\end{tabular}

Segundo a literatura, estudos realizados com a $M$. oleifera como coagulante primário relatam que a utilização deste extrato bruto não é adequada para sistemas de abastecimento de água de grande porte. Madrona et al. (2012) e Valverde et al. (2013a) obtiveram resultados de remoção de cor aparente e turbidez com tempo de sedimentação (TS) de 60 min e $120 \mathrm{~min}$, utilizando extrato de $M$. oleifera salina e aquosa, respectivamente. Para diminuir esse tempo de sedimentação, Bratby (2006) sugere adicionar um polímero orgânico como auxiliar de coagulação, a fim de melhorar o desempenho de floculação no que diz respeito à remoção de matéria orgânica, densidade e sedimentação dos flocos. Ravikumar e Sheeja (2012) afirmam que o TS para o pó das sementes de $M$. oleifera é mais longo quando comparado à utilização da associação dos coagulantes $M$. oleifera e sulfato de alumínio (Valverde et al., 2013b).

Em vista dos resultados obtidos, o TS de 15 min foi escolhido por ser o TS padrão normalmente usado nos decantadores das ETAs e por diminuir o tempo total do tratamento de água, quando comparado a resultados da $M$. oleifera como único coagulante.

Considerando-se os resultados obtidos com a aplicação de $30 \mathrm{rpm}(16,1$ $\mathrm{s}^{-1}$ ) (VML) ao processo de coagulação/ floculação, observou-se que o TS está na faixa de $45 \mathrm{~min}$ ou $60 \mathrm{~min}$. De acordo com Ndabigengesere e Narasiah (1996), baixas VML não produzem energia suficiente para manter a formação dos flocos e promover oportunidade de colisão suficientes.

Deste modo, as seguintes condições de operação poderiam ser utilizadas sem diminuição da eficiência do processo: $100 \mathrm{rpm}\left(98,1 \mathrm{~s}^{-1}\right), 105 \mathrm{rpm}\left(105,6 \mathrm{~s}^{-1}\right)$ ou $110 \mathrm{rpm}\left(113,2 \mathrm{~s}^{-1}\right)$ durante $1 \mathrm{~min}$; ou 105 rpm, $110 \mathrm{rpm}$ durante $3 \mathrm{~min}$ para propiciar a mistura rápida; $45 \mathrm{rpm}(29,6$ $\mathrm{s}^{-1}$ ) durante $15 \mathrm{~min}$ para propiciar a mistura lenta; e 15 min para a etapa de sedimentação, em todos os casos citados.

Baghvand et al. (2009) destacam que, o tempo de mistura é um fator importante que deve ser investigado para se obter uma melhor visão sobre o seu papel para a otimização do processo. Avaliando-se os resultados obtidos em relação aos TMR, 1 min é mais viável por ser o menor tempo de mistura estudado.

Em relação à velocidade de mistura rápida (VMR), $100 \mathrm{rpm}$ é mais interessante, por se tratar da menor intensidade de agitação aplicada ao processo, o que está relacionado com a diminuição dos custos com energia. Segundo Julio et al. (2008), quanto maior o gradiente de velocidade, maiores serão os custos de implantação e operação em uma ETA.

As Figuras 1, 2 e 3 apresentam os resultados dos ensaios realizados para o TS de 15 min, em relação às médias das 
eficiências de remoção de cor aparente, turbidez e $\mathrm{UV}_{254 \mathrm{~nm}}$.

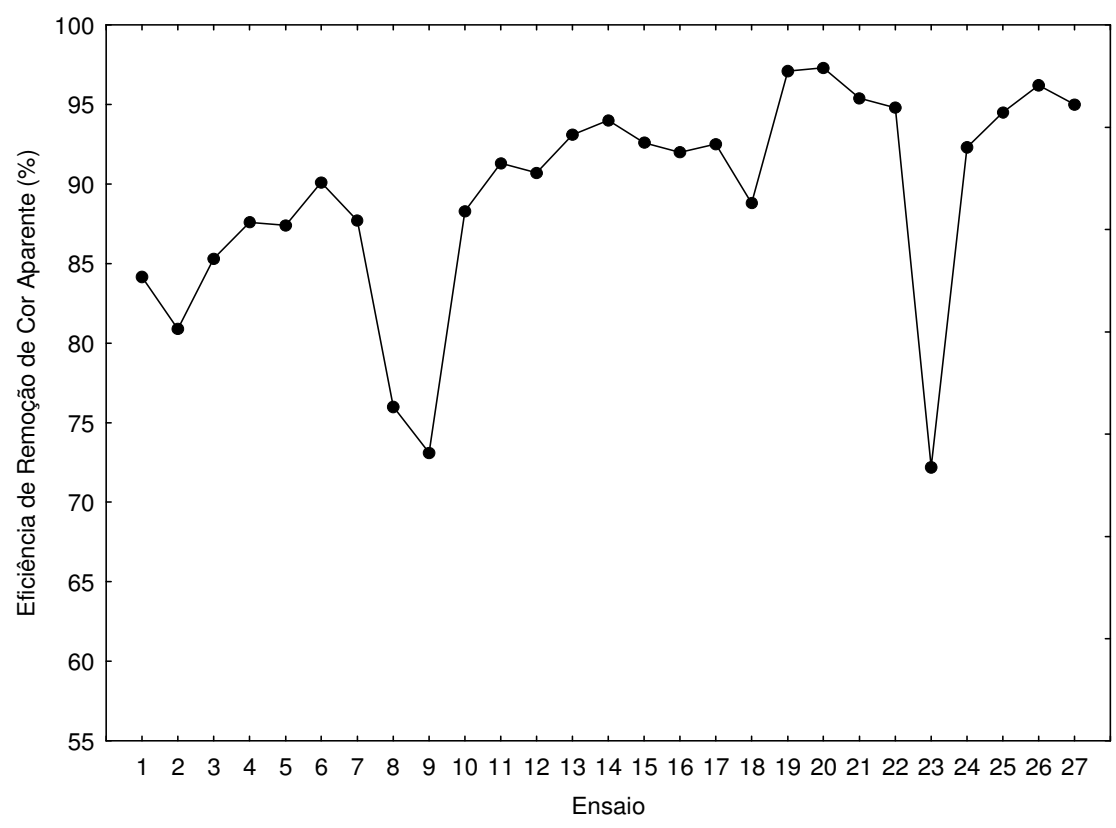

Figura 1. Eficiência de Remoção do Parâmetro Cor Aparente na Associação

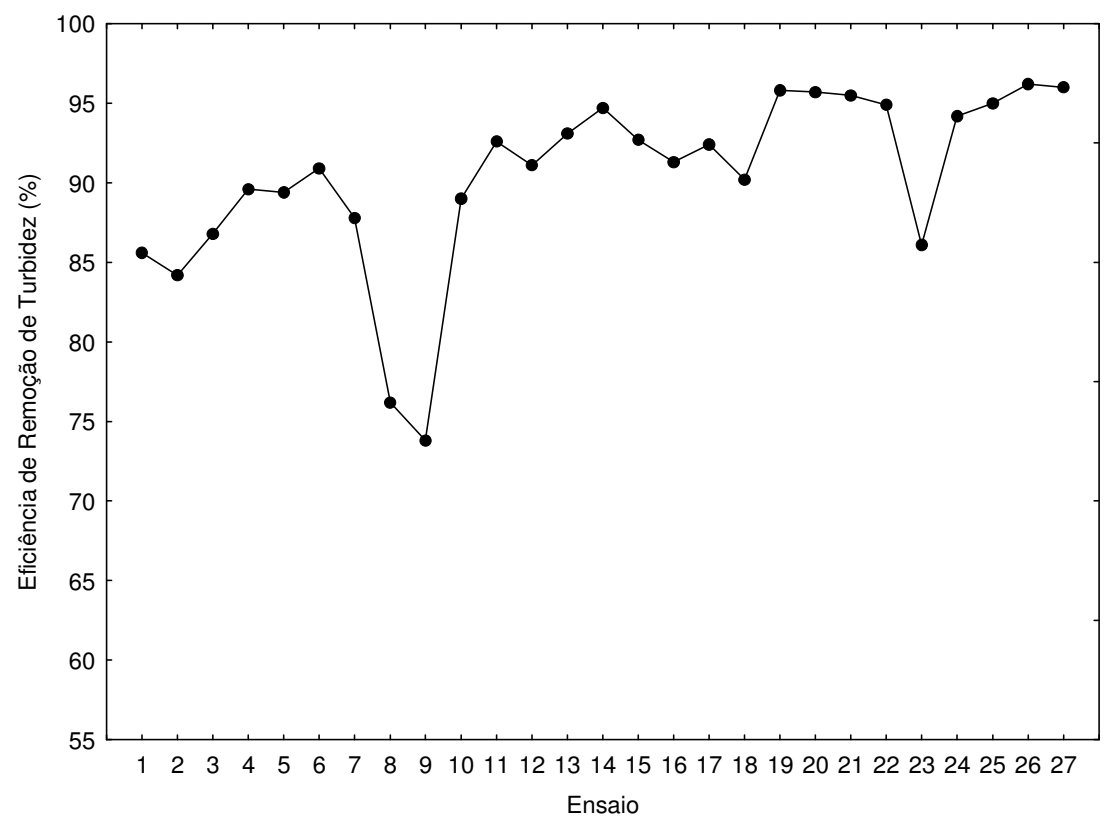

Figura 2. Eficiência de Remoção do Parâmetro Turbidez na Associação 


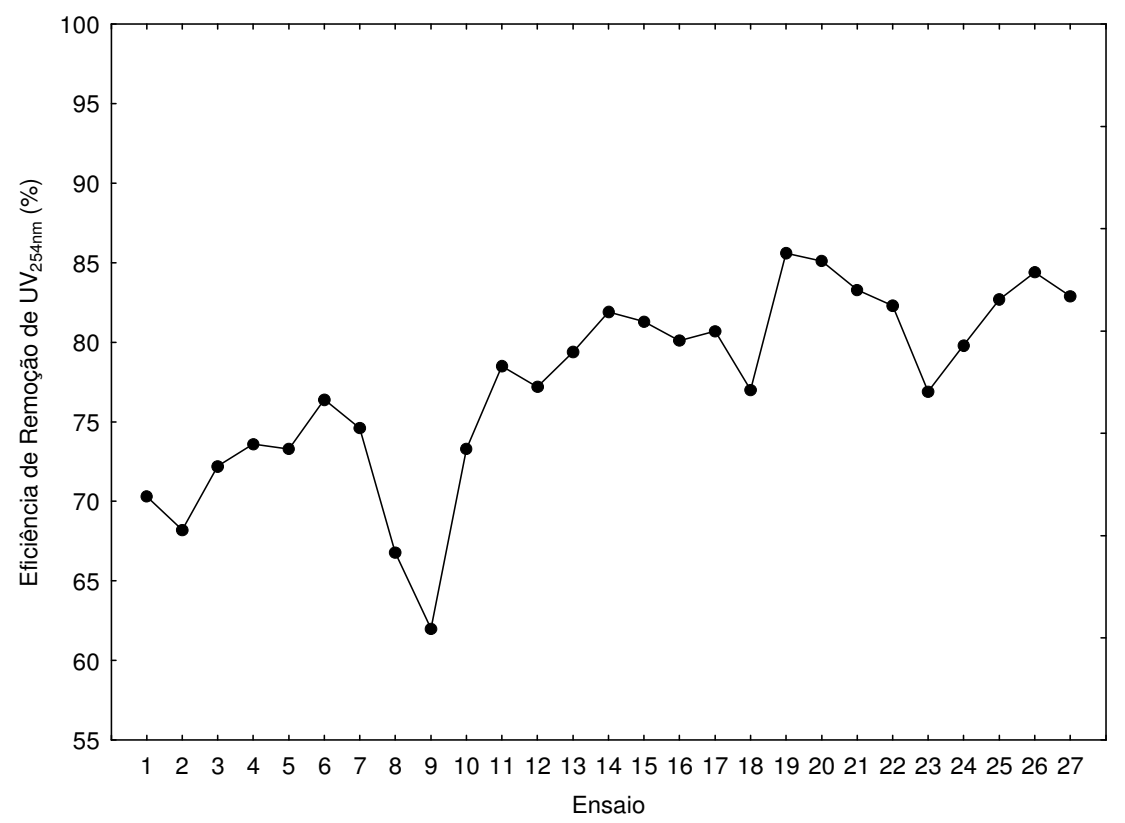

Figura 3. Eficiência de Remoção do Parâmetro $U_{254 n m}$ na Associação

Avaliando-se os resultados obtidos para os três parâmetros de qualidade estudados, as condições de operação ótimas para a associação PAC e M. oleifera são: $100 \mathrm{rpm}$ (VMR), $1 \mathrm{~min}$ (TMR), $45 \mathrm{rpm}$ (VML), $15 \mathrm{~min}$ (TML e TS).

\section{CONCLUSÃO}

Pode-se afirmar com este trabalho que os parâmetros de mistura rápida e lenta, incluindo o tempo e intensidade da mistura, afetam a eficiência de remoção dos parâmetros de qualidade cor aparente, turbidez e $\mathrm{UV}_{254 \mathrm{~nm}}$, no processo de coagulação/floculação e sedimentação.

A associação dos coagulantes PAC e $M$. oleifera contribui para a diminuição do tempo de sedimentação, sendo $15 \mathrm{~min}$ suficientes para a remoção dos parâmetros de qualidade.

Foram obtidos residuais em torno de $12 \mathrm{uH}$ para cor aparente, 3 NTU para turbidez e $0,040 \mathrm{~cm}^{-1}$ para $\mathrm{UV}_{254 \mathrm{~nm}}$, nas condições de operação ótimas: $100 \mathrm{rpm}$ (VMR), 1 min (TMR), 45 rpm (VML) e $15 \min$ (TML).

\section{AGRADECIMENTOS}

Os autores agradecem à Capes e $\mathrm{CNPq}$ pelo suporte financeiro, a UFS pelas sementes de $M$. oleifera e a
SANEPAR pelas amostras de água fornecidas.

\section{REFERÊNCIAS}

ABALIWANO, J. K., GHEBREMICHAEL, K. A., \& AMY, G. L. 2008. Application of the purified Moringa oleifera coagulant for surface water treatment. WaterMill Working Paper Series, n. 5, p. 1-19.

ALI, E. N., MUYIBI, S. A., SALLEH, H. M., ALAM, M. Z., \& SALLEH, M. R. 2010. Production of natural coagulant from Moringa oleifera seed for application in treatment of low turbidity water. Journal Water Resource and Protection, 2, 259-266.

AMAGLOH, F. K., \& BENANG, A. 2009. Effectiveness of Moringa oleifera seed as coagulant for water purification. African Journal of Agricultural Research, 4, 119-123.

APHA. American Public Health Association. 1995. Standard methods for the examination for water and wastewater. $19^{\text {th }}$ ed. Washington.

BAGHVAND, A., ZAND, A. D., MEHRDADI, N., \& KARBASSI, A. 2010. Optimizing coagulation process for low to high turbidity waters using 
aluminum and iron salts. American Journal of Environmental Sciences, 6, 442-448.

BINA, B., MEHDINEJAD, M. H., DALHAMMER, G., RAJARAO, G., NIKAEEN, M., \& ATTAR, H. M. 2010. Effectiveness of Moringa oleifera coagulant protein as natural coagulant aid in removal of turbidity and bacteria from turbid waters. World Academy of Science, Engineering and Technology, 43, 618620.

BRATBY, J. 2006. Coagulation and flocculation in water and wastewater treatment. $2^{\text {th }}$ ed. London, UK: IWA Publishing.

CARDOSO, K. C., BERGAMASCO, R., COSSICH, E. S., \& MORAES, L. C. K. 2008. Otimização dos tempos de mistura e decantação no processo de coagulação/floculação da água bruta por meio da Moringa oleifera Lam. Acta Scientiarum Technology, 3, 193-198.

FATOMBI, K. J., AHOYO, T. A., NONFODJI, O., \& AMINOU, T. 2012. Physico-chemical and bacterial characteristics of groundwater and surface water quality in the lagbe town: Treatment essays with Moringa oleifera seeds. Journal of Water Resource and Protection, 4, 1001-1008.

JOSHUA, R., \& VASU, V. 2013. Characteristics of stored rain water and its treatment technology using Moringa seeds. International Journal of Life Sciences Biotechnology and Pharma Research, 2, 155-174.

JULIO, M. DE, FIORAVANTE, D. A., \& OROSKI, F. I. 2008. Avaliação da influência dos parâmetros de mistura rápida, floculação e decantação no tratamento de água empregando o sulfato de alumínio e o PAC. Ciências Exatas e da Terra, Agrárias e Engenharias, 14109-120.

MADRONA, G. S., BRANCO, I. G., SEOLIN, V. J., ALVES FILHO, B. DE
A., FAGUNDES-KLEN, M. R., \& BERGAMASCO, R. 2012. Evaluation of extracts of Moringa oleifera Lam seeds obtained with $\mathrm{NaCl}$ and their effects on water treatment. Acta Scientiarum Technology, 34, 289-293.

MADRONA, G. S., SERPELLONI, G. B., SALCEDO, A. M., NISHI, L., CARDOSO, K. C., \& BERGAMASCO, R. 2010. Study of the effect of saline solution on the extraction of the Moringa oleifera seeds active component for water treatment. Water, Air and Soil Pollution, 211, 409-415.

MAKKI, H. F., AL-ALAWY, A. F., ADBUL-RAZAQ, N. N., \& MOHAMMED, M. A. 2010. Using aluminum refuse as a coagulant in the coagulation and flocculation processes. Iraqi Journal of Chemical and Petroleum Engineering, 11, 15-22.

NDABIGENGESERE, A., \& NARASIAH, K. S. 1996. Influence of operating parameters on turbidity removal by coagulation with Moringa oleifera seeds. Environmental Technology, 17, 1103-1112.

NWAIWU, N. E, ZALKIFUL, M. A., \& RAUFU I. A. 2012. Seeking an alternative antibacterial and coagulation agent for household water treatment. Journal of Applied Phytotechnology in Environmental Sanitation, 1, 1-9.

POUMAYE, N., MABINGUI, J., LUTGEN, P., \& BIGAN, M. 2012. Contribution to the clarification of surface water from the Moringa oleifera: Case M'Poko river to Bangui, Central African Republic. Chemical Engineering Research and Design, 90, 2346-2352.

PRITCHARD, M., CRAVEN, T., MKANDAWIRE, T., EDMONDSON, A. S., \& O'NEILL, J. G. 2010. A comparison between Moringa oleifera and chemical coagulants in the purification of drinking water - An alternative sustainable solution for 
developing countries. Physics and Chemistry of the Earth, 35, 798-805.

RAVIKUMAR, K., \& SHEEJA, A. K. 2012. Water clarification using Moringa oleifera seed coagulant. In: International Conference on Green Technology, 64-70, India.

RODRÍGUEZ-NÚÑEZ, J. R., SÁNCHEZ-MACHADO, D. I., LÓPEZCERVANTES, J., NÚÑEZGASTÉLUM, J. A., SÁNCHEZDUARTE, R. G., \& CORREAMURRIETA, M. A. 2012. Moringa oleifera seed extract in the clarification of surface waters. International Journal of Environmental Protection, 2, 17-21.

VALVERDE, K. C., MORAES, L. C. K., BONGIOVANI, M. C., CAMACHO, F. P., \& BERGAMASCO, R. 2013a. Coagulation diagrams using the Moringa oleifera Lam and the aluminium sulphate, aiming the removal of color and turbidity of water. Acta Scientiarum Technology, 5, 485-489.

VALVERDE, K. C., COLDEBELLA, P. F., \& BERGAMASCO, R. 2013b. Otimização das condições de operação no processo de clarificação da água superficial por meio da associação dos coagulantes Moringa oleifera Lam e cloreto férrico. Periódico Eletrônico Fórum Ambiental da Alta Paulista, 9, 6576.

VIJAYRAGHAVAN, G, SIVAKUMAR, T, \& VIMAL KUMAR, A. 2011. Application of plant based coagulants for waste water treatment. International Journal of Advanced Engineering Research and Studies, 1, 88-92. 